\title{
A Case of Disseminated Nocardiosis Secondary to the Skin Nodules in an Elderly Woman
}

\author{
Seung Gyun In, M.D., Sung Hyup Han, M.D., Jeong Hyun Shin, M.D., \\ Gwang Seong Choi, M.D., Moon Hyun Chung, M.D. ${ }^{1}$ \\ Department of Dermatology and ${ }^{1}$ Internal Medicine, \\ Inha University College of Medicine, Incheon, Korea
}

\begin{abstract}
Nocardiosis refers to a locally invasive or disseminated infection associated with the Nocardia species. Most infections enter through the respiratory tract and then disseminate systemically. Rarely can a primary nocardial infection of the skin spread to contiguous structures or disseminate to other internal organs in immunocompromised hosts. We describe a 70-year-old woman who suffered from recurrent nodular skin lesions on her right hand, forearm and elbow following inoculation of a traumatic injury. Analysis of the purulent exudates obtained from the nodule revealed Nocardia species. After 20 days, a chest X-ray showed newly developed multiple nodules in both lungs. The diagnosis of systemic nocardiosis was established, and we treated this case with trimethoprim-sulfamethoxazole.
\end{abstract}

(Ann Dermatol (Seoul) 20(2) 82 85, 2008)

Key Words: Disseminated nocardiosis, Nocardia, Nocardiosis

\section{INTRODUCTION}

Nocardiosis is an uncommon infectious disease that can be divided into systemic and cutaneous types ${ }^{1}$. Cutaneous nocardiosis is characterized by one of four clinical manifestations: (i) mycetoma; (ii) lymphocutaneous infection; (iii) superficial skin infections such as cellulitis, abscesses, ulcers or granulomas; and (iv) disseminated infection with cutaneous involvement ${ }^{1-4}$. Here, we present a case of primary cutaneous nocardiosis with dissemination to the lungs.

\section{CASE REPORT}

A previously healthy 70-year-old woman presented

Received July 2, 2007

Accepted for publication February 5, 2008

Reprint request to: Gwang Seong Choi, M.D., Department of Dermatology, Inha University Hospital, 7-206, Sinheung-dong 3-ga, Jung-gu, Incheon 400-711, Korea. Tel: 82-32-890-2238, Fax: 82-32-890-2236, E-mail: garden@ inha.ac.kr with recurrent painful skin lesions on the right arm for three months. She remembered a minor scratch of her hand that had been caused by contaminated scissors. Examination of the skin revealed multiple erythematous nodules on the right dorsum of the hand, forearm and elbow (Fig. 1). Abnormal laboratory test results included a total white blood cell count of 12,900 cells $/ \mathrm{mm}^{3}$ with $91 \%$ neutrophils. No abnormal findings were revealed by the chest X-ray, electrocardiograph, blood biochemistry or urine examination. In addition, the gram stain and potassium hydroxide $(\mathrm{KOH})$ preparations of the pus sample smear were reported negative. A biopsy of the skin showed granulomatous inflammation with central suppuration, but did not demonstrate any fungal elements despite special stainings (PAS, methenamine silver stain) (Fig. 2). Sporotrichosis was suspected and an initial treatment with oral itraconazole for 3 weeks resulted in no significant improvement. Following that, an oral potassium iodide was used for 2 weeks, but no improvement was shown.

During antifungal medication, the patient complained of severe general weakness and the chest X-ray taken after admission showed newly developed 

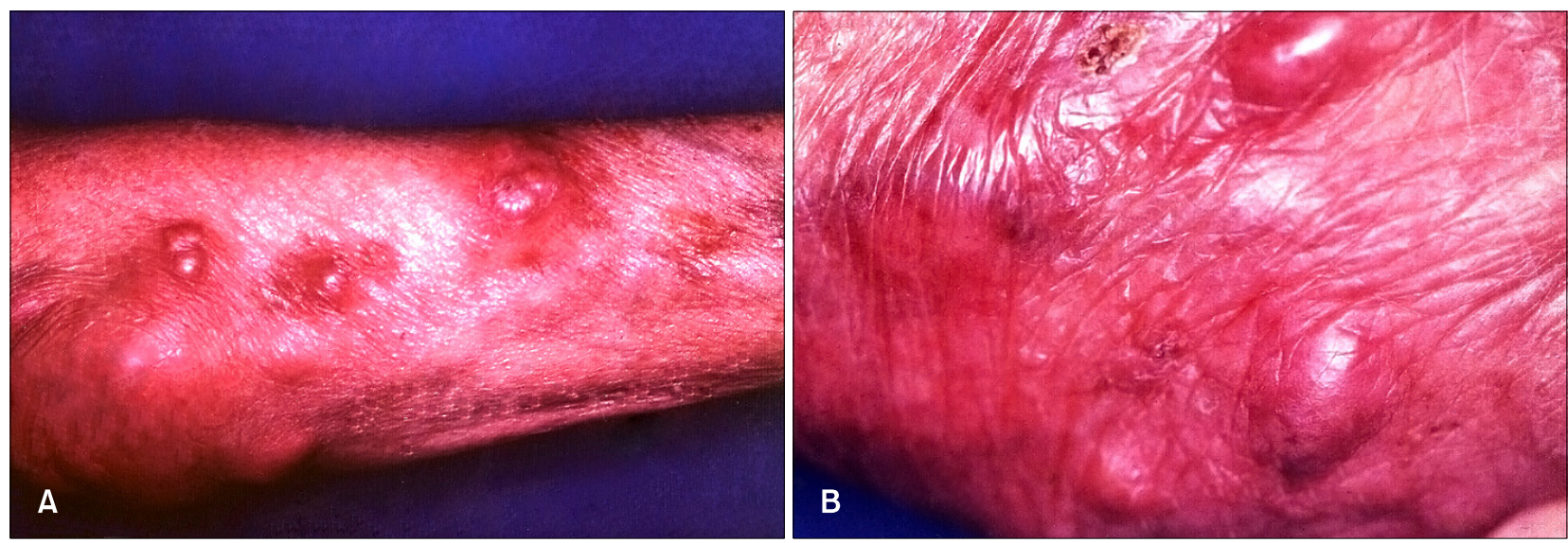

Fig. 1. Multiple erythematous nodules on the right forearm (A) and the dorsum of hand (B).
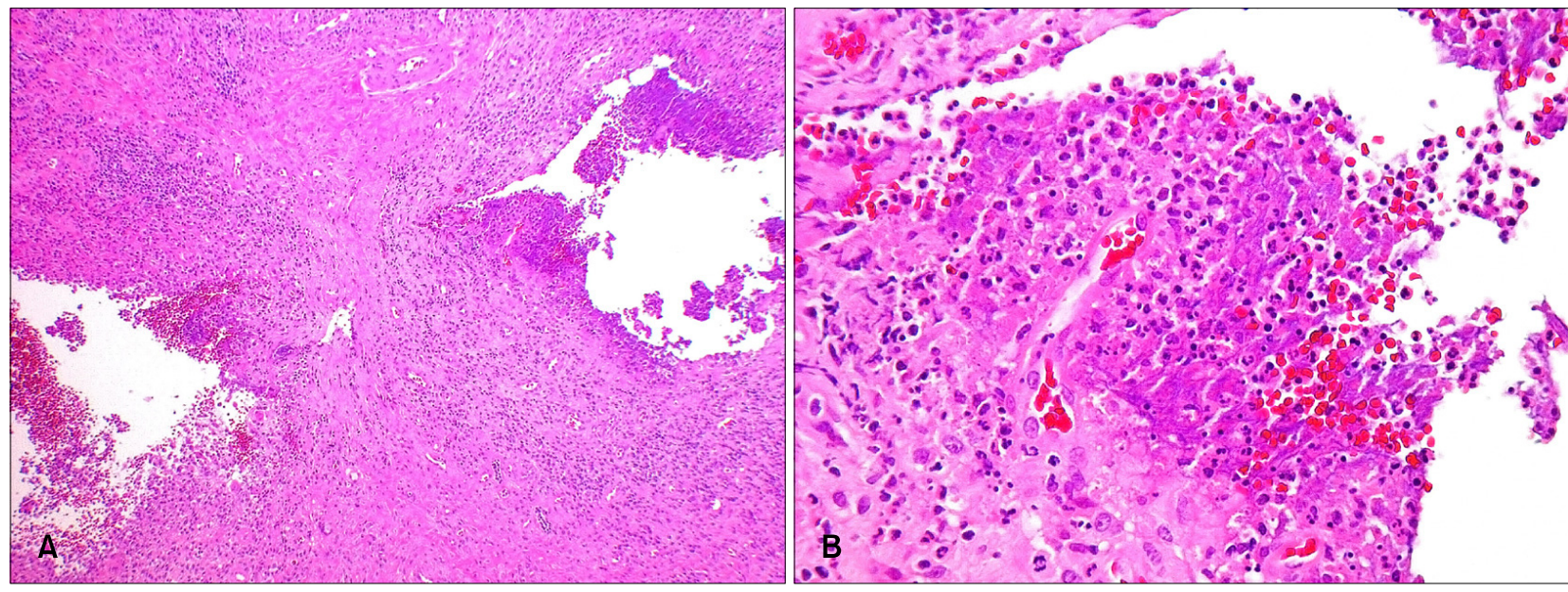

Fig. 2. Granulomatous inflammation with central suppurative inflammation (A: H\&E, $\times 40 ; B: H \& E, \times 400$ ).

multiple nodules in both lungs (Fig. 3). A chest computed tomography showed an opportunistic lung infection.

The purulent discharge from erythematous nodule of the hand, cultured on potato dextrose agar, showed typical colonies of Nocardia species (Fig. 4A). Microscopically, the cultured organisms were gram-positive (Fig. 4B), weakly acid-fast stained with a modified Ziehl-Neelsen stain. Under the diagnosis of nocardiosis, treatment with oral trimethoprimsulfamethoxazole was started and continued for 4 months. The patient responded to the treatment, including complete resolution of the pulmonary nodules and cutaneous abscesses. Only residual scars remained after 4 months of therapy.

\section{DISCUSSION}

Nocardia species are aerobic, filamentous, grampositive and partially acid-fast actinomycetes found in the soil; they cause systemic and cutaneous infections and usually cause an opportunistic infection in immunocompromised patients ${ }^{1-4}$. Primary cutaneous nocardiosis comprises only 5 percent of nocardial infections ${ }^{5}$. Cutaneous nocardiosis is characterized by one of the four clinical manifestations.

Mycetoma is a chronic, indurated, granulomatous and tumour-like mass with draining sinuses localized at the site of injury, and the most common cutaneous manifestation of $N$. brasiliensis ${ }^{1,2}$. Lymphocutaneous infection, also known as sporotrichoid infection, usually begins as an ulcerated papule at 
the site of injury, and is followed by advancing lymphangitis and subcutaneous nodules along the lymphatic drainage. Most lesions initially involve the hand or forearm, and patients usually have a history of a puncture wound and/or a history of farm or garden work ${ }^{2,3}$. The causative organism is usually $N$. brasiliensis ${ }^{1}$. The third form is comprised of superficial skin infections such as ulcers, granulomas, cellulitis and abscesses, and the causative organism is either N. brasiliensis or N. asteroides. The fourth, a cutaneous manifestation of nocardial

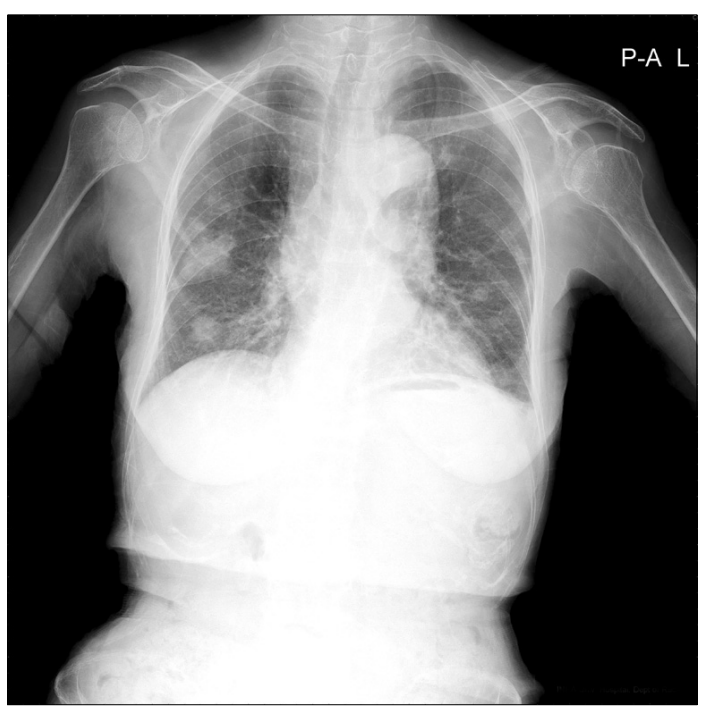

Fig. 3. Newly developed multiple nodules in both lungs. infection, is a disseminated systemic infection with secondary skin involvement and is usually associated with $N$. asteroides ${ }^{1,2}$. The type of infection following inoculation depends on the immune status of the host and the strain of Nocardia ${ }^{4}$. The diagnosis can be confirmed by cultural isolation of the organism and biochemical characteristics ${ }^{6}$.

Primary cutaneous nocardiosis usually does not disseminate, although there have been a few cases of dissemination in both immunocompetent and immunocompromised patients ${ }^{5,7-9}$. The lungs and brain are the primary target organs for dissemination? ${ }^{\text {? }}$ This patient had no underlying disease, but it was suggested that her immunity was affected by old age.

Treatment of nocardiosis usually involves a combined medical and surgical approach. Sulphonamides are the drugs of choice in nocardial infections, with a combination of trimethoprim and sulfamethoxaxole seemingly superior to sulphonamides alone. Minocycline and amikacin as well as imipenem are other choices ${ }^{1-7}$. The suggested duration of therapy ranges from 6 weeks for minor infections to 1 year for severe systemic diseases ${ }^{1-3,5,7}$. But the duration of treatment is still controversial ${ }^{4,6}$. Treatment failure occurs in up to 20 percent of patients with disseminated disease and mortality in nocardemic patients is $44 \sim 85$ percent, emphasizing the need for the prompt recognition of this serious infection ${ }^{5}$.

This is the first case of primary cutaneous nocar-
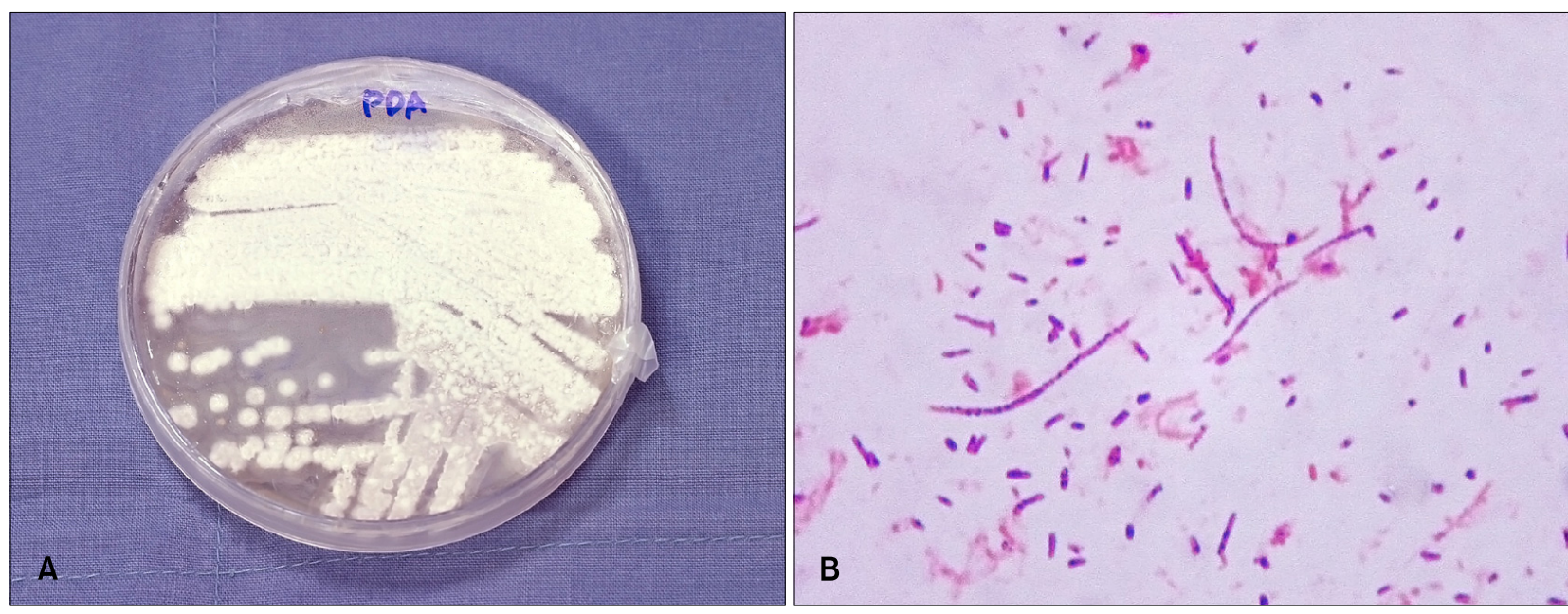

Fig. 4. (A) Whitish, wrinkled, heaped up colonies on potato dextrose agar plate cultured for 43 days. (B) Gram stain showed gram-positive branching organisms $(\times 100)$. 
diosis with dissemination to the lungs emerged in Korea.

\section{REFERENCES}

1. Kalb RE, Kaplan MH, Grossman ME. Cutaneous nocardiosis. Case reports and review. J Am Acad Dermatol 1985;13:125-133.

2. Karakayali G, Karaarslan A, Artuz F, Alli N, Tekeli A. Primary cutaneous Nocardia asteroides. $\mathrm{Br} \mathrm{J}$ Dermatol 1998;139:919-920.

3. Lee SH, Suh CW, Choi JH, Sung KJ. A case of primary cutaneous sporotrichoid nocardiosis caused by Nocardia asteroides. Ann Dermatol 1999;11: 90-93.

4. Aydingoz IE, Candan I, Dervent B, Hitit G. Primary cutaneous nocardiosis associated with intra- articular corticosteroid injection. Int J Dermatol 2001;40:196-198.

5. George SJ, Rivera AM, Hsu S. Disseminated cutaneous nocardiosis mimicking cellulitis and erythema nodosum. Dermatol Online J 2006;12:13.

6. Paredes BE, Hunger RE, Brassthen LR, Brand CU. Cutaneous nocardiosis caused by Nocardia brasiliensis after an insect bite. Dermatology 1999;198: 159-161.

7. Wlodaver CG, Tolomeo T, Benear JB II. Primary cutaneous nocardiosis mimicking sporotrichosis. Arch Dermatol 1988;124:659-660.

8. Boixeda P, Espana A, Suarez J, Buzon L, Ledo A. Cutaneous nocardiosis and human immunodeficiency virus infection. Int J Dermatol 1991;30:804-805.

9. Kahn FW, Gornick CC, Tofte RW. Primary cutaneous Nocardia asteroides infection with dissemination. Am J Med 1981;70:859-863. 